\section{The use of olive oil for skin health in a Saudi population: A cross-sectional study}

\author{
Faisal Alnemer, ${ }^{1}$ Raed Aljohani, ${ }^{1}$ \\ Abdulrahman Alajlan, ${ }^{1}$ \\ Mohammad Aljohani, ${ }^{1}$ \\ Ibrahim Alozaib, ${ }^{1}$ Emad Masuadi, ${ }^{2,3}$ \\ Aamir Omair, 2,3 \\ Mohammed I. Al Jasser ${ }^{1-3}$ \\ ${ }^{1}$ Division of Dermatology, College of \\ Medicine, King Saud bin Abdulaziz \\ University for Health Sciences, Riyadh; \\ ${ }^{2}$ Research Unit, College of Medicine, \\ King Saud bin Abdulaziz University for \\ Health Sciences, Riyadh; ${ }^{3}$ King \\ Abdullah International Medical \\ Research Center, Riyadh, Saudi Arabia
}

\section{Abstract}

The use of topical olive oil (OO) for skin health is common among Saudis and worldwide. Therefore, this cross-sectional study is aimed at assessing the use of topical OO for skin health among Saudis in Riyadh, Saudi Arabia. The questionnaire was administered to Saudis at four different malls in Riyadh, Saudi Arabia. A total of 401 participants were enrolled in the study. The results showed that the average knowledge score of the participants was $3.4 \pm 0.3$ (out of 5) and $87 \%$ had fair knowledge. The top source of knowledge among the participants was friends/relatives. In the attitude section, the average score was $4.1 \pm 0.5$ (out of 5). Females had a higher attitude score $4.2 \pm 0.5$ as compared to males $4 \pm 0.5$ $(\mathrm{P}=0.03)$. The most common reason for using topical $\mathrm{OO}$ among the participants was skin moisturizing (73\%). Female gender was the only significant factor associated with the use of topical OO $(\mathrm{P}<0.001)$. The use of topical OO for skin health is highly prevalent in Riyadh, Saudi Arabia. Therefore, more awareness of the benefits and adverse effects of topical $\mathrm{OO}$ use is required especially from health educational organizations. The preliminary results of this study suggest further research with a larger sample in an academic setting across the nation.

\section{Introduction}

The skin is one of the most important sites of manifestation of diseases. The consequences of poor skin health are not only limited to the physiological health, but it also extends to the psychological wellbeing. Although conventional medicine is the main treatment nowadays, complementary and alternative medicine (CAM) is becoming a popular option for the treatment of skin diseases. Olive oil (OO) is widely used among people worldwide for skin health. It is composed mainly of fatty acids and other minor components. ${ }^{1}$ The fatty acids are mainly subdivided into unsaturated (oleic and linoleic acids) and saturated types (palmitic and stearic acids). The other minor components include $\alpha$-tocopherol, phenol compounds. ${ }^{1}$ Olive oil phenols can be divided into three categories: simple phenols, secoiridoids, and lignans, all of which inhibit auto-oxidation. Major phenols include hydroxytyrosol, tyrosol, oleuropein, and ligstroside. ${ }^{2}$ This unique composition is what makes $\mathrm{OO}$ have features such as anti-inflammatory, antioxidant and antimicrobial properties. ${ }^{3}$

The studies on the use of OO for skin health are limited. The results of these studies vary from beneficial to harmful. Because of the multiple properties of $\mathrm{OO}$, it is potentially important to assess the use of OO for skin health. This might provide us with a deeper look at the significance and potentially different applications of OO. We have observed that $\mathrm{OO}$ is commonly used in Saudi Arabia; however, studies evaluating this subject are lacking. Therefore, our aim in this study is to assess the knowledge, attitude, and practice of topical OO use for skin health among Saudis in Riyadh, Saudi Arabia.

\section{Materials and methods}

The study has been performed according to the declaration of Helsinki principles and was approved by the institutional ethics committee at King Abdullah International Medical Research Center (KAIMRC) (IRBC/1576/18).

This cross-sectional study was conducted at four shopping malls during the period from February 2019 to March 2019 in Riyadh. An informed consent was obtained from all enrolled subjects. All Saudis with or without skin conditions were included in the study, while non-Saudis and illiterate people were excluded. The questionnaire was developed in Arabic and has two parts. The first part was intended to gather the demographic characteristics. These variables included gender, age, marital status, academic qualification, occupation, income, and source of knowledge. The second part of the questionnaire consisted of 33 questions divided into three sections which were the knowledge (14 questions),
Correspondence: Faisal Khalid Alnemer, Division of Dermatology, College of Medicine, King Saud bin Abdulaziz University for Health Sciences, P.O. Box 3660, Riyadh 11481, Saudi Arabia.

E-mail: Alnemer467@ksau-hs.edu.sa

Key words: Olive oil; Complementary and alternative medicine; Skin; Dermatology.

Contributions: The authors contributed equally.

Conflict of interest: The authors declare no potential conflict of interest.

Funding: None.

Ethical approval: Reviewed and approved by the institutional ethics committee at King Abdullah International Medical Research Center (IRBC/1576/18).

Availability of data and material: Data and materials are available by the authors.

Please cite this article as: Alnemer F, Aljohani $R$, Alajlan A, et al. The use of olive oil for skin health in a Saudi population: A cross-sectional study. Dermatol Rep 2022;14:9364.

Received for publication: 25 August 2021. Accepted for publication: 4 September 2021.

This work is licensed under a Creative Commons Attribution-NonCommercial 4.0 International License (CC BY-NC 4.0).

(C) Copyright: the Author(s), 2022

Licensee PAGEPress, Italy

Dermatology Reports 2022; 14:9364

doi:10.4081/dr.2022.9364

attitude (9 questions), and practice (10 questions). The knowledge and attitude questions were scored using the five-point Likert scale ranging from "strongly agree" to "strongly disagree". To formulate both the average knowledge and attitude scores, the five-point Likert scale values were used ranging from 1-5. The average score for each participant was calculated by adding up the score of every question and dividing by the number of questions. Furthermore, to calculate the average scores for the whole study population, the average scores for every participant in each section were summed up and divided by the number of participants. To make sure that the questionnaire is answered carefully and diligently, negative questions have been added to the knowledge section. Questions 2, 10, 11, 14 are presented as wrong statements about $\mathrm{OO}$ and its use. The five-point Likert scale values in these questions are reversed, which means "strongly agree" is equal to 1 
and "strongly disagree" is equal to 5. Based on the knowledge score, the study population was categorized further into poor (1-2), fair (3-4), and excellent (5). This helps in classifying whether the majority of the population has poor, fair, or excellent knowledge.

The questionnaire was assessed by two dermatologists and one faculty member from the research unit at College of Medicine, King Saud bin Abdulaziz University for Health Sciences, for content validity. The questionnaire was then pilot tested twice. The first testing was done on 50 participants, and the second testing was done on 30 participants, to assess the clarity and time required for completion. Modifications on the questionnaire were made accordingly. The Cronbach's alpha values for both the knowledge and attitude sections were 0.674 and 0.746 , respectively.

The sample size was estimated to be 385. It was calculated with the assumption that $50 \%$ of the population use OO for skin health, with a $95 \%$ confidence level and 5\% margin of error. Convenient sampling was done, and to adjust to no responses, approximately an additional $5 \%$ were added to the sample size to become 401 .

Data analysis was done using the Statistical Package for the Social Sciences version 20 (SPSS In., Chicago, IL, USA). The descriptive statistics including frequencies and percentages were used to describe the categorical variables. The mean and standard deviation were generated for the numerical data. The univariate and multivariate logistic regression analyses were used to test the relationship between the $f$ topical $\mathrm{OO}$ and the demographics. A test with a $P$-value of $\leq 0.05$ was considered statistically significant.

\section{Results}

The total number of the participants was 401. The mean age of the participants was $27.7 \pm 8.4$ years and the majority were females (73.5\%). Of the participants, $54.5 \%$ had an education level of a bachelor's degree or higher. Moreover, $80.3 \%$ were employed and $10.3 \%$ were students (Table 1). Out of the 401 participants, $356(88.7 \%)$ have used topical OO. Moreover, 343 (96.3\%) participants who have used topical OO had a positive experience. With univariate analysis, the use of $\mathrm{OO}$ was more in females $(\mathrm{P}<0.001)$ and those with lower income $(\mathrm{P}=0.002)$ (Table 2).

Multivariate regression analysis

Table 1. Subject characteristics $(n=401)$.

\begin{tabular}{lccc}
\hline Variable & Categories & n & \\
Age (years) Mean \pm SD & & $27.7 \pm 8.4$ & 26.5 \\
Gender & Male & 106 & 73.5 \\
\hline Marital status & Female & 295 & 65.3 \\
& Single & 262 & 34.7 \\
Education & Married & 139 & 1 \\
& Elementary & 4 & 4.5 \\
& Intermediate & 18 & 40 \\
& High school & 161 & 51 \\
& Bachelor & 205 & 2.5 \\
& Masters & 10 & 1 \\
\hline Occupation & PhD & 4 & 80.3 \\
& Employee & 321 & 2.3 \\
& Freelance & 9 & 5.5 \\
& Unemployed & 22 & 10.3 \\
& Student & 41 & 1.6 \\
Income (Saudi Riyals) & Other - Not salaried & 7 & 67.6 \\
& $<5000$ & 269 & 21.1 \\
& $5000-10000$ & 84 & 9.5 \\
& $10000-20000$ & 38 & 1.8 \\
\hline Olive oil use & $>20000$ & 7 & 88.7 \\
& Yes & 356 & 11.3 \\
\hline & No & 45 &
\end{tabular}

showed that female gender was the only factor associated with the use of topical OO (Table 3). 67 (18.8\%) participants used topical OO for skin only, 53 (14.9\%) for hair only, and $236(66.3 \%)$ for both. The most common reason for using topical OO among the participants was skin moisturizing $(73 \%)$, brittle hair $(56.2 \%)$, and hair fall $(41.3 \%)$ (Figure 1). OO was also commonly used for eczema and acne (14.9\% and 6.7\%, respectively). $40.1 \%$ of the participants used $\mathrm{OO} \geq$ once per week over the past 12 months (Figure 2).

In the knowledge section, the average knowledge score of the participants was $3.4 \pm 0.3$ (out of 5 ). The analysis showed that $20(5 \%)$ of the participants had excellent knowledge, 349 (87\%) had fair knowledge, 
and $32(8 \%)$ had poor knowledge. The top sources of knowledge among the participants were friends/relatives (52.6\%), social media $(32.6 \%)$, and the internet $(22.5 \%)$. Only $11.4 \%$ acquired knowledge from a physician. $90.1 \%$ of the participants agreed that topical OO can help moisturize the skin. $65.4 \%$ of the participants believe that topical OO has a potential to cure skin diseases. Moreover, $47.2 \%$ stated that using topical $\mathrm{OO}$ is beneficial due to its antiinflammatory effect. Furthermore, $41.5 \%$ of the participants consider topical OO effective in skin lightening. Only $11.1 \%$ believe that topical OO can be harmful on children due to skin irritations (Table 4).

In the attitude section, the average attitude score was $4.1 \pm 0.5$ (out of 5). Females had a higher attitude score $4.2 \pm 0.5$ as compared to males $4 \pm 0.5(\mathrm{P}=0.03)$. The attitude of the participants toward topical OO use was generally positive; $89.8 \%$ of the participants agree that topical $\mathrm{OO}$ is beneficial for skin health, and $78.2 \%$ agree based on personal experience. Furthermore, $92 \%$ of the participants agree that topical OO is beneficial as it is mentioned in Quran and Sunnah. Only $7.5 \%$ agree that topical OO is not beneficial due to its lack of medical reliability (Table 5).

$51.7 \%$ of the participants did not mix $\mathrm{OO}$ with anything. The others used it with honey $(20.3 \%)$, blackseed $(17.8 \%)$, and petroleum jelly (11\%). Other mixtures included sugar, ginger, and almond oil. The majority of participants $(79.4 \%)$ started using $\mathrm{OO}$ for skin health more than 1 year ago, the other $(20.6 \%)$ started within 1 year. When asked about where do the participants purchase/obtain OO from, $46.8 \%$ obtained it from perfume stores, $38.9 \%$ from stores that specialize in selling $\mathrm{OO}$ products, and $32.2 \%$ from supermarkets. The most frequent quantity of $\mathrm{OO}$ used topically was 1 teaspoon $(31.5 \%)$, and less frequently 3 or more teaspoons $(25.3 \%), 2$ teaspoons (23.9\%), and $<1$ teaspoon (19.3\%). 41.8\% of the participants use OO mainly before sleeping, 33.3\% after taking a shower, 33\% when symptoms become severe, $4.8 \%$ before taking a shower, and $3.7 \%$ when exposed to the sun.

\section{Discussion}

The use of OO for therapeutic and healing purposes is a well-known part of the Saudi culture. Taking into consideration the high prevalence of OO uses among Saudis and the limited evidence for its benefit in skin health, it is essential to assess the knowledge, attitude, and practice of using
OO for skin health among Saudis. In the present study, $88.7 \%$ of the participants have used topical OO. This is considered higher than any other prevalence mentioned in Saudi CAM studies. The reported preva-

lence of CAM in Saudi Arabia in two studies was $84.6 \%$ and $73 \%$, respectively. ${ }^{4,5}$ On the other hand, the prevalence of CAM use worldwide is notably less. In Australia, the prevalence of CAM use is $48.5 \% .{ }^{6}$ Also, the

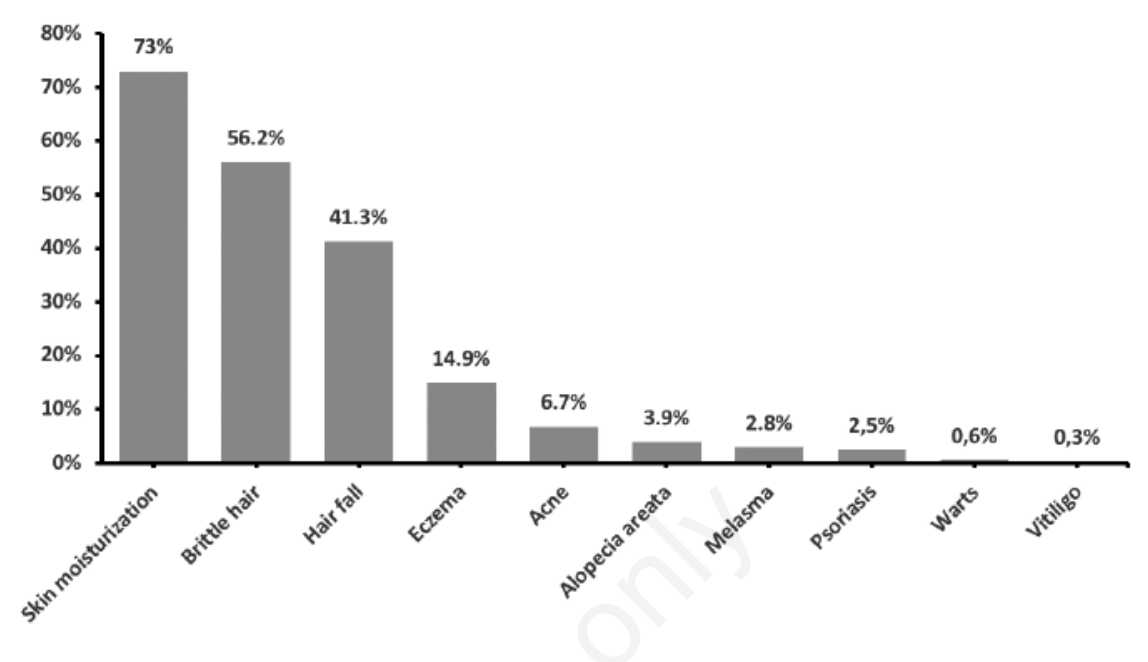

Figure 1. Conditions for which olive oil was used $(n=356)$.

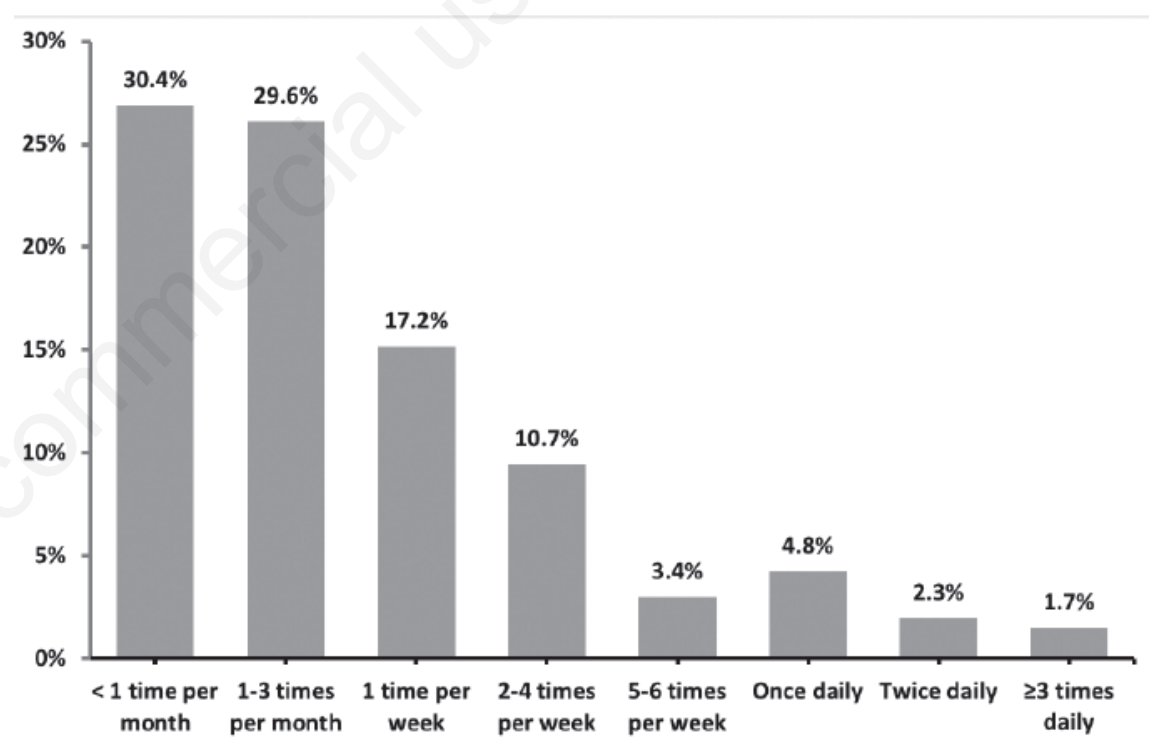

Figure 2. Frequency of topical olive oil application in the past 12 months.

Table 3. Multivariate logistic regression analysis of factors associated with the use of olive oil.

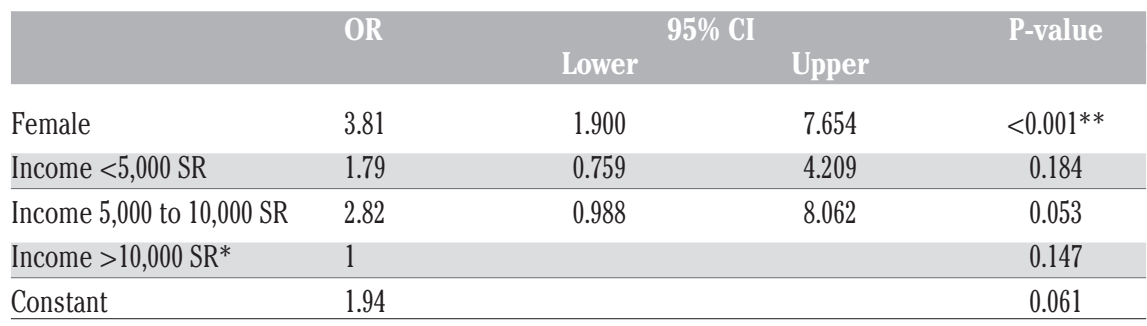

* Reference group; **significant. 
prevalence of CAM use in the United States ranges from $32 \%$ to $54 \%{ }^{7}$ Lastly, in Europe, it has been reported to range from 20 to $50 \%{ }^{8}$ This significant difference could be due to cultural and religious differences.

The majority of our study population had a fair to excellent knowledge on the topical OO use. This could be explained by the religious background, since olive and its derivatives have been mentioned in Quran and Sunnah (Hadith). Moreover, most of the participants were familiar with the topical use of OO since $88.7 \%$ of the participants have used topical OO. The top sources of knowledge were friends/relatives and social media with only a minority who acquired knowledge from a physician. This is in agreement with Elolemy et al., who reported that mass media and family/relative were the main sources of CAM knowledge. ${ }^{4}$ This could be a result of the fact that it is more convenient to obtain medical information from social media and family members rather than from a qualified physician. Another possibility could be the reluctance of mentioning or asking about the use of CAM from patients and physicians, respectively. In multiple studies, more than half of the participants did not discuss CAM with their physicians. ${ }^{4,5}$

Females had a significantly higher attitude score as compared to males. Interestingly, female gender was the only factor associated with the use of topical OO in our study. Some studies have shown a similar trend of more use of CAM among females. ${ }^{7-9}$ This could be explained by the fact that females are more interested in cosmetics and skin care.

The most common reason for using topical OO was skin moisturization. There is conflicting evidence in the literature with regards to the effect of $\mathrm{OO}$ on skin barrier and hydration. In a pilot study, 115 neonates were divided into 3 treatment groups: no oil, OO, and sunflower seed oil. The treatments were applied twice daily on the left side of the body for 4 weeks. Both oil groups had significantly improved hydration, and no significant differences in transepidermal water loss (TEWL). However, there were some alterations in the lipid structure of the skin barrier. ${ }^{10}$ On the other hand, in a controlled study of 19 adults who applied $\mathrm{OO}$ on one forearm, $\mathrm{OO}$ resulted in significant damage to the skin barrier. ${ }^{11}$ This negative effect of $\mathrm{OO}$ on skin barrier could be attributed to a specific component of OO, which is oleic acid. According to a laboratory study on mice, oleic acid might increase the epidermal permeability. ${ }^{12}$ In addition, one study suggests that oleic acid induces inflammation of keratinocytes through $\mathrm{N} \square$ methyl $\square \mathrm{D} \square$ aspartic acid (NMDA) $\square$ type receptors. ${ }^{13}$ Moreover, NMDA receptor antagonists inhibited the increase of TEWL caused by oleic acid. ${ }^{13}$ More studies are required to determine the

Table 4. Knowledge regarding topical olive oil (OO) use.

\begin{tabular}{|c|c|c|c|c|c|c|}
\hline & Question & $\begin{array}{c}\text { Strongly } \\
\text { agree, n (\%) }\end{array}$ & $\begin{array}{l}\text { Agree, } \\
\text { n (\%) }\end{array}$ & $\begin{array}{l}\text { Neutral, } \\
\text { n (\%) }\end{array}$ & $\begin{array}{c}\text { Disagree, } \\
\text { n (\%) }\end{array}$ & $\begin{array}{c}\text { Strongly } \\
\text { disagree, n (\%) }\end{array}$ \\
\hline \multicolumn{2}{|r|}{1 Topical use of 00 contributes in moisturizing the skin } & $184(45.8)$ & $178(44.3)$ & $32(8)$ & $8(2)$ & $0(0)$ \\
\hline \multicolumn{2}{|r|}{2 Topical 00 protects the skin from sun light } & $34(8.6)$ & $63(15.9)$ & $159(40.2)$ & $119(30.1)$ & $21(5.2)$ \\
\hline \multicolumn{2}{|r|}{3 Topical 00 contributes in treating skin diseases } & $82(20.8)$ & $176(44.6)$ & $106(26.8)$ & $28(7.1)$ & $3(0.7)$ \\
\hline \multicolumn{2}{|r|}{ Skin lightening is one of the benefits of topical 00} & $54(13.7)$ & $110(27.8)$ & $133(33.7)$ & $85(21.5)$ & $13(3.2)$ \\
\hline \multicolumn{2}{|r|}{5 Topical 00 is effective for blackheads in the skin } & $27(6.8)$ & $82(20.7)$ & $191(48.2)$ & $89(22.5)$ & $7(1.8)$ \\
\hline \multicolumn{2}{|r|}{ Topical 00 limits the occurrence of wrinkles } & $63(16.2)$ & $150(38.6)$ & $127(32.6)$ & $46(11.8)$ & $3(0.8)$ \\
\hline 7 & Topical 00 contributes in the regeneration of skin cells & $78(19.8)$ & $173(43.9)$ & 108 (27.4) & $30(7.6)$ & $5(1.3)$ \\
\hline \multicolumn{2}{|r|}{ Frequent topical use of $\mathrm{OO}$ causes dryness of the skin } & $21(5.3)$ & $38(9.5)$ & $95(23.9)$ & 187 (47) & 57 (14.3) \\
\hline \multicolumn{2}{|r|}{ Irritation of the skin in children is a disadvantage of topical 00} & $12(3)$ & $32(8.1)$ & $132(33.5)$ & $164(41.5)$ & $55(13.9)$ \\
\hline \multicolumn{2}{|r|}{10 Topical 00 delays wound healing } & $19(4.9)$ & $49(12.5)$ & $178(45.5)$ & 118 (30.2) & $27(6.9)$ \\
\hline \multicolumn{2}{|r|}{11 Topical 00 increases the risk of infection } & $18(4.6)$ & $41(10.5)$ & $142(36.4)$ & $155(39.7)$ & $34(8.8)$ \\
\hline \multicolumn{2}{|r|}{1200 has antioxidant properties } & $54(13.6)$ & $137(34.6)$ & $131(33.1)$ & $63(15.9)$ & $11(2.8)$ \\
\hline \multicolumn{2}{|r|}{1300 has anti-inflammatory properties } & $51(12.9)$ & $135(34.3)$ & 154 (39.1) & $49(12.4)$ & $5(1.3)$ \\
\hline \multicolumn{2}{|r|}{1400 contains carcinogens } & $12(3)$ & $13(3.3)$ & 46 (11.5) & $158(39.5)$ & 171 (42.7) \\
\hline
\end{tabular}

Table 5. Attitude towards the use of olive oil (OO).

\begin{tabular}{|c|c|c|c|c|c|c|}
\hline & Question & $\begin{array}{l}\text { Strongly } \\
\text { agree, } \mathrm{n}(\%)\end{array}$ & $\begin{array}{l}\text { Agree, } \\
\text { n }(\%)\end{array}$ & $\begin{array}{l}\text { Neutral, } \\
\text { n (\%) }\end{array}$ & $\begin{array}{l}\text { Disagree, } \\
\text { n (\%) }\end{array}$ & $\begin{array}{c}\text { Strongly } \\
\text { disagree, } \mathrm{n}(\%)\end{array}$ \\
\hline 1 & Topical 00 is beneficial for skin health & $138(34.5)$ & $221(55.3)$ & $33(8.2)$ & $7(1.8)$ & $1(0.2)$ \\
\hline 2 & Topical 00 is beneficial due to its natural origin & $189(47.8)$ & $180(45.6)$ & $24(6.1)$ & $2(0.5)$ & $0(0)$ \\
\hline 3 & $\begin{array}{l}\text { Topical } 00 \text { is beneficial because it is mentioned in } \\
\text { the Quran and Sunnah }\end{array}$ & $220(55.5)$ & $144(36.5)$ & $27(6.7)$ & $2(0.5)$ & $3(0.8)$ \\
\hline 4 & $\begin{array}{l}\text { Topical } 00 \text { is beneficial because it is commonly } \\
\text { used among the society }\end{array}$ & $128(32.5)$ & $165(41.9)$ & $66(16.7)$ & $31(7.9)$ & $4(1)$ \\
\hline 5 & Topical 00 is beneficial based on personal experience & $133(34.2)$ & $171(44)$ & $59(15.1)$ & $24(6.2)$ & $2(0.5)$ \\
\hline 6 & Society's attitude towards topical 00 is positive & $165(41.2)$ & $169(42.2)$ & $43(10.7)$ & $13(3.2)$ & $11(2.7)$ \\
\hline 7 & $\begin{array}{l}\text { Topical } 00 \text { is not beneficial because I do not believe } \\
\text { in alternative medicine }\end{array}$ & $14(3.5)$ & $15(3.8)$ & $53(13.3)$ & $181(45.5)$ & $135(33.9)$ \\
\hline 8 & Downsides of using topical 00 are more than its benefits & $10(2.5)$ & $18(4.5)$ & $64(16.1)$ & $193(48.5)$ & $113(28.4)$ \\
\hline 9 & Topical 00 is not beneficial due to its lack of medical reliability & $12(3)$ & $18(4.5)$ & $64(16.2)$ & $184(46.3)$ & $119(30)$ \\
\hline
\end{tabular}


effect of olive oil on skin barrier.

The second most common use of $\mathrm{OO}$ was for hair problems. There is limited evidence on the benefit of $\mathrm{OO}$ for hair. Oleuropein (a major component of $\mathrm{OO}$ ) has been shown to increase hair growth and hair follicle size in mice. ${ }^{14}$ One study showed that $\mathrm{OO}$ inhibited the ability of fungi to penetrate the hair follicle. ${ }^{15}$ However, OO use in specific diseases like scalp seborrheic dermatitis should be dealt with caution since its saturated and unsaturated fatty acids may stimulate the growth of Malassezia species. ${ }^{16}$ In addition, OO is used as a standard component of in vitro culture medium for Malassezia species. ${ }^{17}$

One of the limitations of our study is that it represents Riyadh city only. Thus, the results cannot be generalized to the whole country as each region has its different culture and habits. Another limitation is that shopping malls do not fairly represent the society and its different levels especially the economical level. Moreover, the lack of studies assessing knowledge, attitude, and practice related to $\mathrm{OO}$ for skin health in Saudi Arabia and worldwide, makes it difficult to guarantee the credibility of our results. This study did not record those participants who refused to participate and hence the calculation of a response rate was not possible. Furthermore, the questionnaire used for this study is a self-reported questionnaire.

\section{Conclusions}

Our study found that $88.7 \%$ of the participants have used topical OO, with the female gender being the only factor associated with $\mathrm{OO}$ use. There were various reasons for which Saudis use topical OO. The most common reasons were for skin mois- turization and hair problems. This study might serve as basis for future studies on OO use for skin health, as research on this subject is limited.

\section{References}

1. Viola P, Viola M. Virgin olive oil as a fundamental nutritional component and skin protector. Clin Dermatol 2009;27:159-65.

2. Waterman E, Lockwood B. Active components and clinical applications of olive oil. Altern Med Rev 2007;12:33142.

3. Hashmi MA, Khan A, Hanif M, et al. Traditional Uses, Phytochemistry, and Pharmacology of Olea europaea (Olive). Evid Based Complement Alternat Med. 2015;2015:541591.

4. Elolemy AT, Albedah AM. Public knowledge, attitude and practice of complementary and alternative medicine in riyadh region, saudi arabia. Oman Med J 2012;27:20-6.

5. Al-Faris EA, Al-Rowais N, Mohamed $A G$, et al. Prevalence and pattern of alternative medicine use: the results of a household survey. Ann Saudi Med 2008;28:4-10.

6. MacLennan AH, Wilson DH, Taylor AW. Prevalence and cost of alternative medicine in Australia. Lancet 1996;347:569-73.

7. Eisenberg DM, Davis RB, Ettner SL, et al. Trends in alternative medicine use in the United States, 1990-1997: results of a follow-up national survey. JAMA 1998;280:1569-75.

8. Fisher P, Ward A. Complementary medicine in Europe. BMJ 1994;309:107-11.

9. Ni H, Simile C, Hardy AM. Utilization of complementary and alternative medicine by United States adults: results from the 1999 national health interview survey. Med Care 2002;40:353-8.

10. Cooke A, Cork MJ, Victor S, et al. Olive Oil, Sunflower Oil or no Oil for Baby Dry Skin or Massage: A Pilot, Assessorblinded, Randomized Controlled Trial (the Oil in Baby SkincaRE OBSeRvE. Study). Acta Derm Venereol 2016;96:323-30.

11. Danby SG, AlEnezi T, Sultan A, et al. Effect of olive and sunflower seed oil on the adult skin barrier: implications for neonatal skin care. Pediatr Dermatol 2013;30:42-50.

12. Jiang SJ, Zhou XJ. Examination of the mechanism of oleic acid-induced percutaneous penetration enhancement: an ultrastructural study. Biol Pharm Bull 2003;26:66-8.

13. Katsuta Y, Iida T, Hasegawa K, et al. Function of oleic acid on epidermal barrier and calcium influx into keratinocytes is associated with N-methyl D-aspartate-type glutamate receptors. Br J Dermatol 2009;160:69-74.

14. Tong T, Kim N, Park T. Topical Application of Oleuropein Induces Anagen Hair Growth in Telogen Mouse Skin. PLoS One 2015;10:e0129578.

15. Bahuguna S, Kushwaha R. Influence of different oils on penetration of human hair by fungi. Int $\mathrm{J}$ Cosmetic Sci 1993;15:1-5.

16. Kaneko T, Makimura K, Abe M, et al. Revised culture-based system for identification of Malassezia species. J Clin Microbiol 2007;45:3737-42

17. Siegfried E, Glenn E. Use of olive oil for the treatment of seborrheic dermatitis in children. Arch Pediatr Adolesc Med 2012;166:967. 